\title{
The "clover technique" as a novel approach for correction of post-traumatic tricuspid regurgitation
}
0 . Alfieri, MD
M. De Bonis, MD
E. Lapenna, MD
E. Agricola, MD
A. Quarti, MD
F. Maisano, MD

From the Department of Cardiac Surgery, S. Raffaele University Hospital, Milan, Italy.

Received for publication Nov 1, 2002; revisions requested Dec 9, 2002; revisions received Dec 23, 2002; accepted for publication Dec 27, 2002.

Address for reprints: Michele De Bonis, MD, Department of Cardiac Surgery, San Raffaele University Hospital, Via Olgettina 60, 20132, Milano, Italy (E-mail: michele.debonis@hsr.it).

J Thorac Cardiovasc Surg 2003;126:75-9

Copyright (C) 2003 by The American Association for Thoracic Surgery

0022-5223/2003\$30.00+0

doi:10.1016/S0022-5223(03)00204-6
Objective: To describe a novel technique, named "clover," to correct complex post-traumatic tricuspid valve lesions.

Methods: Five patients with severe post-traumatic tricuspid insufficiency underwent valve reconstruction with the clover technique, a new surgical approach that consists of stitching together the middle point of the free edges of the tricuspid leaflets, producing a clover-shaped valve. The mechanism of tricuspid regurgitation was complex in all patients, and right ventricular function was always moderately to severely depressed. An echocardiographic study was performed after cardiopulmonary bypass, at discharge, and at follow-up.

Results: Cardiopulmonary bypass time was $32 \pm 6.3$ minutes and crossclamp time was $23 \pm 7.4$. There was no hospital mortality or morbidity. Intraoperative transesophageal and predischarge transthoracic echocardiography showed perfect results in all patients. No late deaths occurred. At the latest follow-up, extending to 14.2 months (mean 11.3; median 12.4), all patients were asymptomatic (New York Heart Association class I) with trivial ( 2 patients) or no residual regurgitation ( 3 patients) on 2-dimensional echocardiogram. No transvalvular gradient was revealed in any patient. A significant reduction of the right ventricular end-diastolic dimensions was noted as well (from $54 \pm 7.1 \mathrm{~mm}$ to $40 \pm 7.5 \mathrm{~mm}, P<.001$ ).

Conclusions: In this preliminary experience, the clover technique increased the feasibility of tricuspid valve repair in case of severe traumatic tricuspid valve insufficiency, leading to very satisfactory mid-term results even in the presence of complex lesions or dilatation and deterioration of the right ventricle.

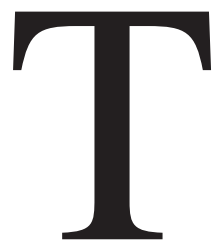

ricuspid regurgitation is a rare complication of blunt chest trauma. This lesion is often not promptly diagnosed because of its subtle clinical manifestations at first and the presence of associated injuries in multiple trauma. Therefore, even though around 120 cases have been reported so far in the literature, its real prevalence is probably underestimated.

The majority of patients described have been treated with valve replacement but, more recently, repair of the native valve is emerging as the treatment of choice.

In 1996 we reported our initial experience in this field, describing 5 cases of post-traumatic tricuspid regurgitation treated with valve repair by means of classic Carpentier's techniques. ${ }^{1}$

In this article we report 5 more cases of valve repair for post-traumatic tricuspid regurgitation performed with a novel technique named "clover," which has not been described previously. 
TABLE 1. Patient data and mechanisms of post-traumatic tricuspid regurgitation

\begin{tabular}{|c|c|c|c|c|c|c|c|c|c|c|}
\hline Patient & Sex & $\begin{array}{c}\text { Age } \\
\text { (years) }\end{array}$ & $\begin{array}{c}\text { Time lapse } \\
\text { from trauma } \\
\text { (years) }\end{array}$ & TR & AD & $\begin{array}{l}\text { Chordal } \\
\text { rupture }\end{array}$ & $\begin{array}{c}\text { Papillary } \\
\text { rupture }\end{array}$ & ALP & PLP & $\begin{array}{c}\text { RVEDD } \\
(\mathrm{mm})\end{array}$ \\
\hline 1 & $\mathrm{~F}$ & 75 & 25 & $4+$ & ++ & ++ & & ++ & ++ & 63 \\
\hline 2 & $M$ & 27 & 6 & $4+$ & ++ & & anterior & ++ & ++ & 44 \\
\hline 3 & $M$ & 31 & 5 & $4+$ & ++ & ++ & & ++ & ++ & 52 \\
\hline 4 & $\mathrm{M}$ & 42 & 12 & $4+$ & ++ & & anterior & ++ & & 53 \\
\hline $5^{*}$ & $\mathrm{M}$ & 35 & 11 & $4+$ & ++ & ++ & & ++ & & 58 \\
\hline
\end{tabular}

$A D$, Annular dilatation; $A L P$, anterior leaflet prolapse; $P L P$, posterior leaflet prolapse; $S L$, septal leaflet; $T R$, tricuspid regurgitation.

*In patient 5 a posterior and septal leaflet-restricted motion was present as well.

\section{Material and Methods Patients}

From July 2001 to February 2002, 5 patients with post-traumatic tricuspid insufficiency underwent valve reconstruction with the clover technique. All had a history of blunt chest trauma secondary to motor vehicle accident. Thoracic trauma was isolated in one case, while the others had multiple injuries. The interval between trauma and operation ranged from 5 to 25 years. Symptoms included palpitations, dyspnea, and fatigue. Two patients were in New York Heart Association (NYHA) class II and 3 were in class III. The more common physical findings were jugular vein distension, peripheral edema, hepatic congestion, and a systolic murmur that increased with inspiration. On admission the electrocardiogram showed sinus rhythm in all patients. The diagnosis was made in all cases by transthoracic followed by transesophageal Doppler echocardiography.

The preoperative patient data and the mechanism of tricuspid regurgitation are detailed in Table 1 . Some degree of right ventricular overload was present in all patients (mean right ventricular end-diastolic dimension $54 \pm 7.1 \mathrm{~mm}$, range 44 to $63 \mathrm{~mm}$ ). Right ventricular function was moderately to severely depressed in all patients.

\section{Surgical Technique}

A median sternotomy was performed and, after aortobicaval cannulation, cardiopulmonary bypass was instituted at mild hypothermia. The aorta was crossclamped and cold blood cardioplegia was delivered antegradely. The tricuspid valve was exposed trough a conventional oblique right atriotomy. The valve anatomy and mechanism of regurgitation were assessed. In all patients, the anterior leaflet of the tricuspid valve was completely or partially flail as a result of chordal rupture (3 patients) or rupture of the anterior papillary muscle (2 patients). A posterior leaflet prolapse was associated in 3 cases, whereas 1 patient had concomitant restricted motion of the posterior and septal leaflet due to the tethering effect produced by the severe right ventricular dilatation. Annular dilatation was always present.

The new surgical approach that we adopted consists of stitching together the middle point of the free edges of the tricuspid leaflets, producing a clover-shaped valve (Figures 1 and 2). In all cases a 5.0 polipropylene suture without pledgets was used. A Carpentier tricuspid ring (number 36) was always implanted to correct the annular dilatation and deformation and stabilize the repair, preventing further dilatation.
In all cases, following the correction, saline was injected into the right ventricle to test valvular competence. Additionally, during rewarming, the tricuspid valve function was evaluated by transesophageal echocardiography.

\section{Follow-up}

Before discharge, all patients underwent control transthoracic echocardiography. Diuretics were prescribed at discharge together with oral anticoagulation, which was taken for 3 months after the repair. At follow-up, extending to 14.2 months (mean 11.3; median 12.4), all patients were in NYHA class I. All were submitted again to echocardiographic study.

\section{Statistical Analysis}

The results are expressed as means \pm standard deviation. Statistical significance was obtained by 2 -tailed paired $t$ test for continuous variables.

\section{Results}

Intraoperative direct inspection of the tricuspid valve and assessment of the mechanism of regurgitation confirmed the preoperative echocardiographic findings. Cardiopulmonary bypass time was $32 \pm 6.3$ minutes and crossclamp time was $23 \pm 7.4$ minutes. All patients survived the operation and were discharged within 6 days after an uneventful postoperative course. Intraoperative transesophageal and predischarge transthoracic echocardiography showed perfect results in all patients. No late deaths occurred. At the latest follow-up, all patients were asymptomatic (NYHA class I) with trivial (2 patients) or no residual regurgitation (3 patients) on 2-dimensional echocardiogram. No transvalvular gradient was revealed in any patient. A significant reduction of the right ventricular end-diastolic dimensions was noted as well (from $54 \pm 7.1 \mathrm{~mm}$ to $40 \pm 7.5 \mathrm{~mm}, P<.001$ ). All patients returned to their preoperative occupation.

\section{Discussion}

In this article we report our preliminary experience with a new surgical technique, named "clover technique," for repair of traumatic tricuspid valve insufficiency. To the best of our knowledge, the clover technique has not been described 
A

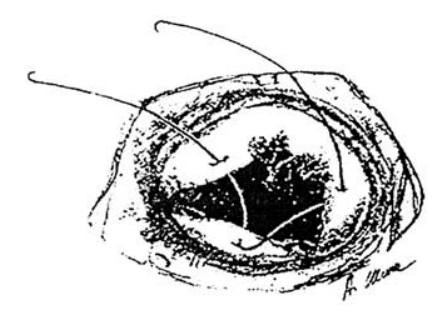

C

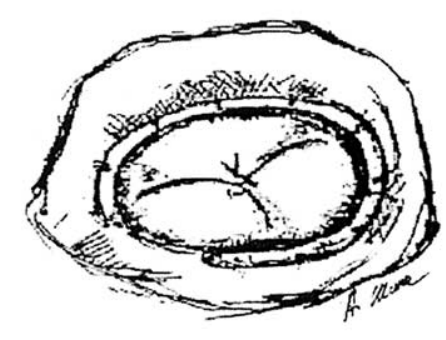

B

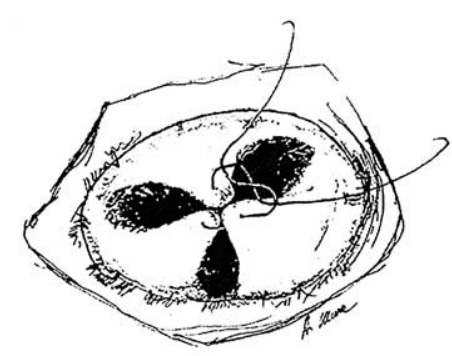

Figure 1. Surgical steps of the clover technique.

previously. In this series, such a novel approach, successfully applied in 5 patients, increased the feasibility of tricuspid valve repair in this difficult setting, leading to very satisfactory mid-term results even in presence of complex lesions or dilatation and deterioration of the right ventricle.

Traumatic tricuspid rupture presumably involves a sudden increase of the right ventricular intracavitary pressure during the isometric systolic phase, when the heart is full and the valve is closed. ${ }^{2}$ The frequency of this disease is probably underestimated because isolated chronic tricuspid regurgitation is usually well tolerated and most patients experience few or no symptoms after trauma. ${ }^{3}$ Because of its subtle clinical course, in most cases progressive dilatation of the tricuspid annulus occurs and right ventricular dilatation and dysfunction eventually develops before surgery.

During the past decade more patients with traumatic tricuspid rupture have benefited from valve repair as a result of improved surgical techniques, widespread use of intraoperative echocardiography, and general agreement that valve repair, when feasible, should be superior to valve replacement. Indeed, in the tricuspid position, both mechanical and biological prostheses have been proven to provide less than satisfactory results with a high incidence of complications. ${ }^{4}$ Considering that patients with post-traumatic tricuspid regurgitation are usually young and active, valve repair seems to be the best option. It avoids prosthesis- related complications and the need for long-term anticoagulation, preserving the geometry and function of the right ventricle.

Traditionally, the operative technique has been dictated primarily by the specific anatomical lesions encountered at the time of surgery. The most frequently reported injury is chordal rupture (55\% of the cases), followed by rupture of the anterior papillary muscle $(27 \%)$ and tear of the leaflets (15\%). Annular dilatation, secondary to right ventricular enlargement, is usually present and contributes to the worsening of the regurgitation. Chordal shortening, sometimes associated with leaflet retraction in the absence of evident rupture, has been described as well.

Several surgical techniques have been used tailored to the specific patient. Artificial chordal implant, quadrangular resection of the flail segment, or chordal transposition have been suggested in presence of chordal rupture, allowing a successful repair in about $45 \%$ of the cases. In presence of papillary muscle rupture, muscle reimplantation was accomplished in $45 \%$ of the reported patients, but significant residual regurgitation after surgery was not unusual. Indeed, the result of papillary muscle rupture repair depends very much on the correct site of reimplantation, which is not always easy to identify considering that a minimal displacement can cause significant distortion of the valve and consequent regurgitation. These conventional reconstructive procedures, besides being technically demanding and some- 


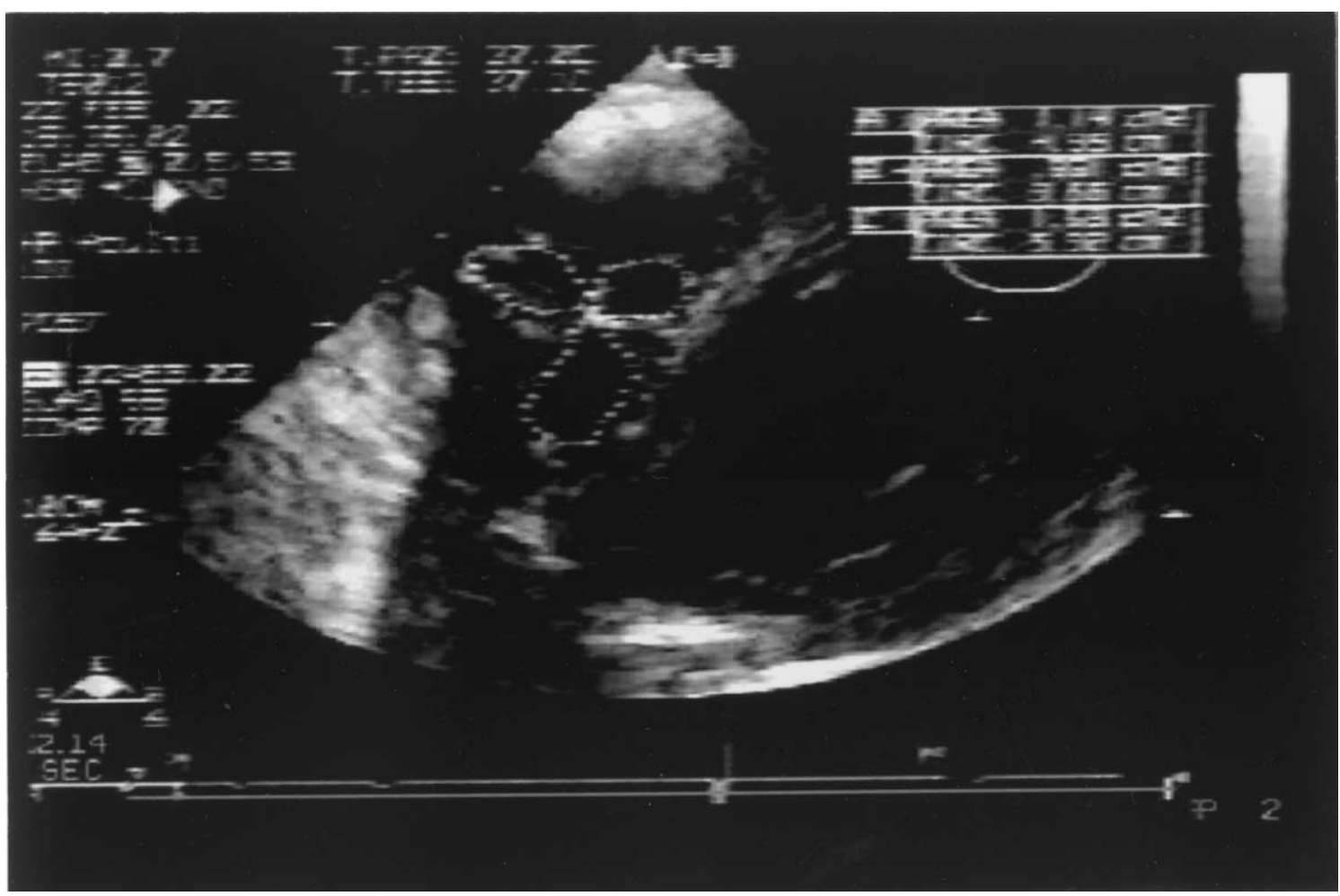

Figure 2. Intraoperative transesophageal view of the clover-shaped tricuspid valve after repair.

times not suitable because of the flimsy nature of the tricuspid apparatus, ${ }^{5}$ often lead to suboptimal results. In traumatic tricuspid regurgitation, the presence of multiple lesions, progressive dilatation, and deterioration of the right ventricle and the contracted state in which the chordae tendineae and the involved leaflets are frequently found tend to preclude valve repair with classic techniques.

Indeed, by using traditional anatomical reconstructive approaches, several unsuccessful attempts at papillary muscle and chordal repair, followed by tricuspid valve replacement, have been reported.

Richard and coworkers ${ }^{6}$ described 9 patients with traumatic tricuspid regurgitation, mainly due to anterior papillary muscle rupture, who underwent surgical correction. Valve repair was performed just in 1 case, whereas the remaining 8 patients had to undergo tricuspid valve replacement. ${ }^{6}$

Holper and colleagues ${ }^{7}$ reported on 5 patients operated on for traumatic tricuspid insufficiency. The mean interval from trauma to surgery in their series was 13.2 years and the mean preoperative NYHA class was 2.9. Intraoperatively all patients showed rupture of the anterior chordae tendineae, 1 patient had multiple ruptures of the leaflets and of the anterior papillary muscle, and all tricuspid valves showed massive annular dilatation. A primary valvular reconstruction was undertaken in 3 patients, of which only 1 was successful. Eventually, 4 tricuspid valves had to be replaced, 3 with a bioprosthesis and 1 with a mechanical valve. $^{7}$

In the Mayo Clinic experience, 13 patients with traumatic tricuspid insufficiency were treated surgically. Repair was considered feasible in 5 cases $(38 \%)$, whereas in the remaining $8(62 \%)$ the valve was replaced. ${ }^{8}$

These data confirm that correction of long-standing posttraumatic tricuspid regurgitation by conventional reconstructive methods is not always feasible or is associated with significant residual valve incompetence. This is consistent with our own previous experience, already reported, ${ }^{1}$ and related to 6 cases of severe post-traumatic tricuspid regurgitation treated by using conventional reconstructive surgical techniques (implant of artificial chordae, papillary muscle reinsertion, commissuroplasty). Some degree of right ventricular overload was present in all patients (mean right ventricular end-diastolic diameter $52 \pm 13.8 \mathrm{~mm}$, range 30 to 60 ). The cardiopulmonary bypass time was 64 \pm 15.4 minutes and the ischemic time $34 \pm 12.7$ minutes. Although tricuspid insufficiency improved after surgery in all patients, at a mean follow-up of $29 \pm 10.1$ months, only 1 patient had no residual regurgitation, whereas valve insufficiency was mild in 2 cases $(1+)$, moderate in $1(2+)$, and moderate-severe $(3+)$ in the remaining 2 . 
We believe, due to the huge right ventricular dilatation resulting from long-standing tricuspid regurgitation, that tethering of the leaflets occurred following anatomical repair, leading to inadequate coaptation.

Because of such suboptimal results, a novel surgical approach, allowing a functional rather than anatomical repair, was conceived and adopted in this context. The application of the clover technique allowed the restoration of a perfect competence of all the tricuspid valves treated, even in presence of complex lesions and huge right ventricular dilatation.

Moreover, this surgical approach seems to be potentially effective in any type of tricuspid insufficiency due to complex mechanisms such as prolapse or flail of multiple leaflets and retractile lesions associated to severe annular dilatation: the perfect results accomplished in 2 more patients with severe tricuspid incompetence due to degenerative disease, recently submitted to this repair in our unit, seem to confirm this impression. We are aware that the small number of patients treated so far does not allow strong statistical support of the effectiveness of this technique and that a longer follow-up is necessary to evaluate the durability of the procedure; nevertheless, in this preliminary series, valvular competence was always restored and the repair was never restrictive. We therefore regard the clover technique as an easy, rapid, and effective approach to correct posttraumatic tricuspid regurgitation and, potentially, as a convenient option in the presence of complex tricuspid valve lesions associated with right ventricular enlargement/dysfunction due to long-standing chronic volume overload.

\section{References}

1. Maisano F, Lorusso R, Sandrelli L, et al. Valve repair for traumatic tricuspid regurgitation. Eur J Cardiothorac Surg. 1996;10:867-73.

2. Naja I, Pomar JL, Barriuso C, Mestrea C, Mulet J. Traumatic tricuspid regurgitation. J Cardiovasc Surg (Torino). 1992;33:256-7.

3. Cahill NS, Beller BM, Linhard JW. Isolated traumatic tricuspid regurgitation. Prolonged survival without operative intervention. Chest. 1972;61:669-72.

4. McGrath LB, Gonzalez Lavin L, Bailey BM, Grunkemeier GL, Fernandez J, Laub GW. Tricuspid valve operations in 530 patients. Twenty-five year assessment of early and late phase events. J Thorac Cardiovasc Surg. 1990;99:124-33.

5. Heulin A, Morin P, Baybion N, Le Feuvre C, Le Pailleur C, Vacheron A. L'insuffisance tricuspidienne d'origine traumatique. Ann Med Interne. 1991;141:285-6.

6. Richard P, Vayre F, Sabouret P, Gandjbakhch I, Ollivier JP. Outcome of traumatic tricuspid insufficiency, treated surgically. Apropos of 9 cases. Arch Mal Coeur Vaiss. 1997;90:451-6.

7. Holper K, Hahnel C, Augustin N, Meisner H. Operative correction of traumatic tricuspid insufficiency. Herz. 1996;21:172-8.

8. Van Son JAM, Danielson GK, Schaff HV, Miller FA. Traumatic tricuspid valve insufficiency. Experience in thirteen patients. $J$ Thorac Cardiovasc Surg. 1994;108:893-8.

\section{Authoritative}

The Journal of Thoracic and Cardiovascular Surgery is the most frequently cited thoracic/cardiovascular surgery journal in the Science Citation Index. An article in JTCVS is cited on average almost twice as often as those in the closest cardiothoracic journal. 